\title{
THE RANGE OF A MEASURE
}

\author{
BY NEIL W. RICKERT ${ }^{1}$
}

Communicated by P. R. Halmos, March 9, 1967

Let $X$ be a measure space, and $\lambda$ a finite measure (countably additive) with values in $R^{n}$. The range of $\lambda$ is the set of all vectors $\lambda(E)$ where $E$ ranges over the measurable subsets of $X$. In a previous paper [2] we introduced a certain positive measure $\nu$, determined by the measure $\lambda$, on the projective space $P^{n-1}$. We then showed that a necessary and sufficient condition that the range of $\lambda$ be a ball is that $\nu$ be an orthogonally invariant measure on $P^{n-1}$. Our purpose in this paper is to show that that result was but a special case of a more general theorem: If $X^{\prime}$ is another measure space, with $\lambda^{\prime}$ a finite measure on $X^{\prime}$ with values in $R^{n}$, and if $\nu^{\prime}$ is the corresponding positive measure on $P^{n-1}$, then a necessary and sufficient condition that the range of $\lambda$ and the range of $\lambda^{\prime}$ have the same convex hull is that (a) $\lambda(X)$ $=\lambda^{\prime}\left(X^{\prime}\right)$ and (b) $\nu=\nu^{\prime}$. If only condition (b) is satisfied, the range of $\lambda$ is a translate of the range of $\lambda^{\prime}$.

The appearance of the convex hull of the range of the measure is not surprising, because, as shown by Liapunoff, if the measure is atom free its range is a convex set (see [2]). The theorem is false if we refer only to the range of the measures, and not to the convex hull of the range, as can easily be shown by example.

For $x$ a point in $S^{n-1}$, we denote by $H_{x}$ the hemisphere $\left\{y \in S^{n-1}\right.$; $\langle y, x\rangle \geqq 0\}$.

Lemma 1. Let $\mu$ be a real Borel measure on $S^{n-1}$. Let $\alpha$ be the natural map of $S^{n-1}$ onto $P^{n-1}$, and let $\nu$ be the Borel measure on $P^{n-1}$ defined by $\nu(E)=\mu\left(\alpha^{-1}(E)\right)$. Then a necessary and sufficient condition that for each $x$ in $S^{n-1}$

$$
\int_{H_{x}}\langle x, y\rangle_{\mu}(d y)=0
$$

is that (a) $\int_{S^{n-1}} y \mu(d y)=0$ and (b) $\nu$ is the zero measure on $P^{n-1}$.

Proof. First assume that (a) and (b) are satisfied. Condition (a) implies that

$$
\int_{H_{x}}\langle x, y\rangle \mu(d y)+\int_{H-x}\langle x, y\rangle \mu(d y)=0
$$

\footnotetext{
${ }^{1}$ Supported by National Science Foundation grant GP-5803.
} 
(note that the integral of $\langle x, y\rangle$ over $H_{x} \cap H_{-x}$ is obviously zero), while condition (b) implies that

$$
\int_{H_{x}}\langle x, y\rangle_{\mu}(d y)-\int_{H-x}\langle x, y\rangle_{\mu}(d y)=0 .
$$

The desired conclusion follows immediately. For the converse assume that for each $x, \int_{H_{x}}\langle x, y\rangle \mu(d y)=0$. It follows immediately that for each $x$

$$
\left\langle x, \int_{S^{n-1}} y \mu(d y)\right\rangle=\int_{H_{x}}\langle x, y\rangle_{\mu}(d y)-\int_{H_{-x}}\langle-x, y\rangle_{\mu}(d y)=0
$$

whence (a) is satisfied. The proof of (b) is similar to the argument used in Lemmas 2 and 3 of [2] so we shall content ourselves with outlining the proof here and leaving the details to the reader. For a fixed $x_{0}$ in $S^{n-1}$, we introduce the function $\phi$ defined on $S^{n-1}$ by $\phi(y)=\max \left\{0,\left\langle x_{0}, y\right\rangle\right\}$. Assuming first that $\mu$ is invariant under the special orthogonal transformations that leave $x_{0}$ fixed it can be readily shown that the hypotheses imply that the convolution of the measure $\mu$ with the function $\phi$ is zero (the convolution being is the sense of convolutions on symmetric spaces). By taking Fourier Stieltjes transforms this implies that the product of the Fourier coefficients of $\phi$ with those of $\mu$ yields zero. But for the spherical functions on $S^{n-1}$ which correspond to spherical functions on $P^{n-1}$, the Fourier coefficients of $\phi$ are not zero, so those of $\mu$ must be zero. This implies (b) for $\mu$ of the special type we are considering. For general $\mu$ we can average its rotations via the orthogonal transformations which fix $x_{0}$, and applying the special case we can conclude that $\int f d \nu=0$ for $f$ a spherical function on $P^{n-1}$. But the hypotheses on $\mu$ are invariant under orthogonal transformations; so it follows that $\int f d \nu=0$ whenever $f$ is an orthogonal transform of a spherical function. But the linear space of continuous functions generated by functions of this type is dense. It follows immediately that $\nu=0$ as required.

LEMma 2. Let $\lambda$ be a finite $R^{n}$-valued measure on the measure space $X$. Then the convex hull of the range of $\lambda$ is determined once we know, for each $x$ in $S^{n-1}, \sup _{E}\langle x, \lambda(E)\rangle$, where $E$ varies over the measurable subsets of $X$.

Proof. The assertion that $r \geqq \sup _{E}\langle x, \lambda(E)\rangle$ is equivalent to the assertion that the range of $\lambda$ is contained in the half space $\{y ;\langle x, y\rangle \leqq r\}$. But the convex hull of the range of $\lambda$ is exactly the intersection of such half spaces, and the result follows. 
Denoting by $|\lambda|$ the total variation measure of $\lambda$, we define $f=d \lambda / d|\lambda|$. Observe that $|f|=1$ almost everywhere, so $f$ defines a map of $X$ into $S^{n-1}$. There are thus induced on $S^{n-1}$ measures $f(\lambda)$ and $f(|\lambda|)$, defined by $f(\lambda)(E)=\lambda\left(f^{-1}(E)\right)$, and $f(|\lambda|)(E)=|\lambda|\left(f^{-1}(E)\right)$ for $E$ a Borel set in $S^{n-1}$. Note that $|f(\lambda)|=f(|\lambda|)$, and that $f(\lambda)(d x)$ $=x f(|\lambda|)(d x)$.

THEOREM 1. The ranges of the measures $\lambda$ and $f(\lambda)$ have the same convex hull.

Proof. Since for $E$ a measurable subset of $X$, and $x$ in $S^{n-1}$, $\langle x, \lambda(E)\rangle \leqq\left\langle x, \lambda\left(f^{-1}\left(H_{x}\right)\right)\right\rangle$, the result follows easily from Lemma 2 .

LEMMA 3. Let $\mu$ and $\mu^{\prime}$ be finite positive measures on $S^{n-1}$, and let $\nu$ and $\nu^{\prime}$ be the corresponding measures induced on $P^{n-1}$ via the natural map $\alpha$. Let $\lambda$ and $\lambda^{\prime}$ be the vector measures on $S^{n-1}$ defined by $\lambda(d x)=x \mu(d x)$ and $\lambda^{\prime}(d x)=x \mu^{\prime}(d x)$. Then a necessary and sufficient condition that the ranges of $\lambda$ and $\lambda^{\prime}$ have the same convex hull is that (a) $\lambda\left(S^{n-1}\right)=\lambda^{\prime}\left(S^{n-1}\right)$ and (b) $\nu=\nu^{\prime}$.

Proof. From Lemma 2 we conclude that the ranges of $\lambda$ and $\lambda^{\prime}$ have the same convex hull if and only if for each $x$ in $S^{n-1}$

$$
\sup _{E}\langle x, \lambda(E)\rangle=\sup _{E}\left\langle x, \lambda^{\prime}(E)\right\rangle \text {. }
$$

That is, if and only if for each $x,\left\langle x, \lambda\left(H_{x}\right)\right\rangle=\left\langle x, \lambda^{\prime}\left(H_{x}\right)\right\rangle$ or, equivalently,

$$
\int_{H_{x}}\langle x, y\rangle \mu(d y)=\int_{H_{x}}\langle x, y\rangle \mu^{\prime}(d y) .
$$

Thus, applying Lemma 1 to the measure $\mu-\mu^{\prime}$, the result easily follows.

LEMMA 4. Let $\mu$ be a finite positive measure on $S^{n-1}$, and let $\nu$ be the induced measure on $P^{n-1}$. Define the measure $\lambda$ by $\lambda(d x)=x \mu(d x)$. Then there is a finite positive measure $\mu^{\prime}$ on $S^{n-1}$ such that (i) $\mu^{\prime}$ induces the same measure $\nu$ on $P^{n-1}$. (ii) If $\lambda^{\prime}$ is defined by $\lambda^{\prime}(d x)=x \mu^{\prime}(d x)$, then $\lambda^{\prime}\left(S^{n-1}\right)=0$. (iii) The convex hull of the range of $\lambda^{\prime}$ is a translate of the convex hull of the range of $\lambda$.

Proof. First consider the case where $\mu$ (and hence $\lambda$ ) is atom free. In this case there is a Borel set $F$ such that $\lambda(F)=\frac{1}{2} \lambda\left(S^{n-1}\right)$. We define $\mu^{\prime}$ by $\mu^{\prime}(E)=\mu(E)-\mu(E \cap F)+\mu(E \cap(-F)$ ) (where here $-F$ means the set of points $\{-y ; y \in F\})$. It is easily seen that $\lambda^{\prime}(E)$ 
$=\lambda(E)-2 \lambda(E \cap F)$. Thus (ii) is true. From the definition of $\mu^{\prime}$, (i) is evidently true. For $E$ a Borel set, denote by $E^{\prime}$ the symmetric difference of $E$ with $F$. It is easy to calculate that $\lambda^{\prime}\left(E^{\prime}\right)=\lambda(E)-\lambda(F)$ and $\lambda^{\prime}(E)=\lambda\left(E^{\prime}\right)-\lambda(F)$. Thus (iii) is satisfied.

Next we consider the case where $\mu$ is completely atomic. In this case we can find a sequence of points $x_{i}(i=1,2, \cdots)$ in $S^{n-1}$ such that $x_{2 i}=-x_{2 i-1}$ and such that $\mu$ has mass $m_{i}$ at $x_{i}$, where $0 \leqq m_{i}, \sum m_{i}$ $<\infty$, and $m_{2 i-1}$ and $m_{2 i}$ are not both zero. We define the measure $\mu^{\prime}$ to have mass $m_{i}^{\prime}$ at $x_{i}$, where $m_{2 i-1}^{\prime}=m_{2 i}^{\prime}=\frac{1}{2}\left(m_{2 i-1}+m_{2 i}\right)$. Then (i) and (ii) are evidently satisfied. If $\left\{r_{i}\right\}$ is a sequence of real numbers with $0 \leqq r_{i} \leqq 1(i=1,2, \cdots)$ we define a sequence $\left\{r_{i}^{\prime}\right\}$ so that $0 \leqq r_{i}^{\prime} \leqq 1 \quad(i=1,2, \cdots)$ and $r_{2 i-1}^{\prime}-r_{2 t}^{\prime}=\left(m_{2 i-1}+m_{2 i}\right)^{-1}\left(2 m_{2 i-1} r_{2 i-1}\right.$ $\left.-2 m_{2 i} r_{2 i}+m_{2 i}-m_{2 i-1}\right)$. It is not difficult to show that such a choice is possible, and that every sequence $\left\{r_{i}^{\prime}\right\}$ with $0 \leqq r_{i}^{\prime} \leqq 1(i=1,2, \cdots)$ can be obtained in this way from a suitable sequence $\left\{r_{i}\right\}$. Now for every point $\sum r_{i} x_{i} m_{i}$ in the convex hull of the range of $\lambda$, the point $\sum r_{i}^{\prime} x_{i} m_{i}^{\prime}=\sum r_{i} x_{i} m_{i}-\frac{1}{2} \lambda\left(S^{n-1}\right)$ is in the convex hull of the range of $\lambda^{\prime}$. Thus (iii) is satisfied.

In the case of a general finite positive measure $\mu$ we proceed simply by writing it as a sum of an atom free measure and a completely atomic measure, and applying the result to each part separately. Evidently (i) and (ii) will be satisfied, and since the range of a measure is the algebraic sum of the range of its nonatomic and completely atomic parts, (iii) will also be satisfied.

Theorem 2. Let $X$ be a measure space, and $\lambda$ a finite countably additive measure on $X$. Set $f=d \lambda / d|\lambda|$, and let $\nu$ be the measure on $P^{n-1}$ defined by $\nu(E)=|\lambda|\left(f^{-1} \alpha^{-1}(E)\right)$ for $E$ a Borel subset of $P^{n-1}$. Let $X^{\prime}$ be another measure space, $\lambda^{\prime}$ a finite countably additive measure on $X^{\prime}$ with values in $R^{n}$, and let the measure $\nu^{\prime}$ on $P^{n-1}$ be analogously defined. Then in order that the convex hull of the range of $\lambda$ and the convex hull of the range of $\lambda^{\prime}$ be identical, it is necessary and sufficient that (a) $\lambda(X)$ $=\lambda^{\prime}\left(X^{\prime}\right)$ and (b) $\nu=\nu^{\prime}$. Condition (b) is satisfied if and only if the convex hull of the range of $\lambda^{\prime}$ is a translate of the convex hull of the range of $\lambda$.

Proof. This now follows easily from Theorem 1 and Lemmas 3 and 4 .

\section{REFERENCES}

1. P. R. Halmos, The range of a vector measure, Bull. Amer. Math. Soc. 54 (1948), 416-421.

2. N. W. Rickert, Measures whose range is a ball, Pacific J. Math. (to appear).

INSTITUTE FOR AdvaNCEd STUdy AND

YALE UNIVERSITY 